\section{Internal iliac artery aneurysm-a cause of leg swelling and cellulitis}

\author{
N Bhasin MRCS SM Jones MBChB J Patel FRCR \\ D Kessel FRCR I Robertson FRCR \\ D C Berridge FRCS D J A Scott FRCS
}

J R Soc Med 2004;97:483-484

Internal iliac artery aneurysms, rare but potentially fatal, can be mistaken for more common medical disorders.

\section{CASE HISTORIES}

\section{Case 1}

A man of 56 had for five weeks been troubled by swelling and cellulitis of the left leg, unresponsive to antibiotics. His medical history included rheumatic fever, hypercholesterolaemia, hypertension, insulin-dependent diabetes and smoking. His body mass index was 40. Venous duplex was normal but CT revealed a $5 \mathrm{~cm}$ saccular aneurysm of the left internal iliac artery with compression of the left ureter and pelvic veins. The patient declined surgery but accepted coil embolization (Cook UK Ltd, Letchworth, UK) of the aneurysm (Figure 1). On venography the aneurysm was seen to be compressing a $5 \mathrm{~cm}$ segment of the left external iliac vein with no venous collateral flow; therefore he was started on anticoagulants to prevent venous thrombosis. At repeat venography six weeks later the findings were identical, so anticoagulation was continued. Six months postoperatively the patient reported intermittent leg swelling, and CT showed a 95\% stenosis of the external iliac vein with a thrombosed internal iliac artery aneurysm. The stenosis was treated with a WallStent (Boston Scientific Europe Ltd, St Albans, UK) and anticoagulation was continued (Figure 2). After a further year there had been no more episodes of cellulitis or leg swelling, though the patient did report mild buttock claudication. Venous duplex showed that the stent was patent without restenosis.

\section{Case 2}

A man of 69 was assessed in the coronary care unit having become short of breath with chest pain and swelling of the

Department of Vascular and Endovascular Surgery, St James's University Hospital, Beckett Street, Leeds LS9 7TF, UK

Correspondence to: Neeraj Bhasin, Vascular Laboratory, 1st Floor, Lincoln Wing, Department of Vascular Surgery, St James's University Hospital, Leeds LS9 7TF, UK

E-mail: neerajbhasin@hotmail.com (a)

(b)

(c)

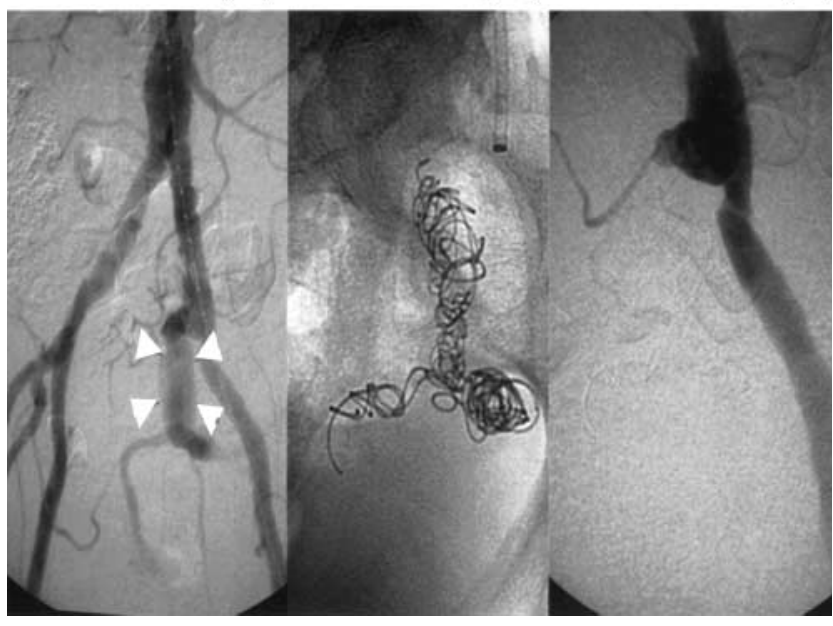

Figure 1 Internal iliac artery embolization, case 1. (a) Arrows indicate aneurysm; (b) coil deployment; (c) completion angiogram showing exclusion of aneurysm

lower limbs after a plane flight. Myocardial infarction was excluded but he was thought to have heart failure. His medical history included peripheral vascular disease, myocardial infarcts, hypertension and an abdominal aortic aneurysm repair. He had a 50-pack-year history of cigarette smoking.

On referral to the vascular team he was found to be anaemic with a pyrexia of $37.7^{\circ} \mathrm{C}$. He had scrotal oedema, a raised jugular venous pressure and basal crackles. His legs were oedematous to the mid thigh, worse on the right side, and there were no palpable pulses distal to the right femoral and left popliteal arteries. Both feet were warm and well perfused. An additional finding was a swollen abdomen, tender to deep palpation in the right iliac fossa. This pain had started during the admission and was associated with

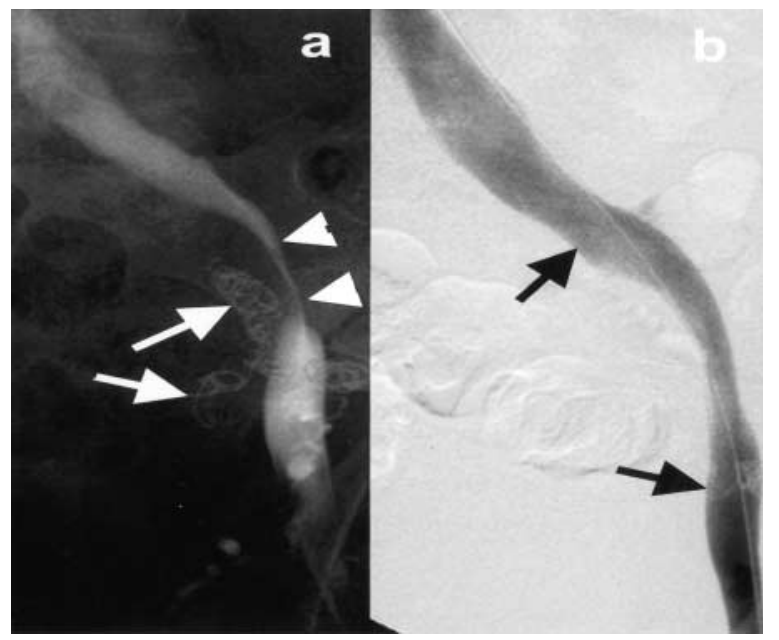

Figure 2 Stenting of external iliac vein, case 1. (a) Arrows point to coils within the aneurysm and vein stenosis; $(b)$ arrows indicate length and position of stent 
watery diarrhoea. The patient also had positive blood cultures for Streptococcus bovis - an organism often associated with gastrointestinal malignancy. He was referred to the general surgical team for further investigation. An ileofemoral deep vein thrombosis was suspected and this was confirmed with duplex. CT, done to investigate the abdominal pain and diarrhoea, revealed a $6.5 \mathrm{~cm}$ right internal iliac artery aneurysm, with no evidence of rupture, compressing the right iliac venous system. Thrombus was noted in the right common iliac vein and inferior vena cava. A filter was placed in the inferior vena cava and the right internal iliac artery aneurysm was treated by coil embolization.

At six months the lower limb oedema had completely resolved. Despite endoscopic and radiographic examinations the cause of the anaemia was never found.

\section{COMMENT}

Internal iliac artery aneurysms make up less than $0.5 \%$ of all intra-abdominal aneurysms. ${ }^{1}$ Because of their rarity and their depth in the pelvis they are difficult to diagnose and tend to present late with symptoms from compression (urological and neurological symptoms, constipation, leg oedema, iliofemoral thrombosis ${ }^{1-3}$ ), expansion, fistulation and rupture. Our two patients developed lower limb oedema due to venous and lymphatic compression, with subsequent cellulitis in one case and deep venous thrombosis in the other. We have not found a previous report of cellulitis as a presenting symptom of an internal iliac artery aneurysm. The natural history of these aneurysms is of expansion and eventual rupture. Rupture is associated with a $58 \%$ mortality. ${ }^{2,4}$ Surgical treatment poses a formidable technical challenge ${ }^{2,4}$ with a mortality of $10 \%$ even in cases treated electively. ${ }^{2}$ Percutaneous coil embolization to thrombose the sac may obviate the need for an open procedure, but only a few case reports are available. ${ }^{3}$ If there is no proximal neck, stent grafts can be used to occlude the aneurysm origin. ${ }^{1}$ Development of venous thrombosis after embolization of an internal iliac artery aneurysm has been reported previously - the result of continued pressure from the embolized aneurysm. ${ }^{2}$ In a separate reported case, a patient was treated by venous thrombectomy, venous stenting and arterial coil embolization. ${ }^{5}$ In our case 2 , venous stenting proved unnecessary.

Internal iliac artery aneurysms should be considered as a potential cause of cellulitis unresponsive to conventional treatment or as a rare cause of lower limb oedema and deep venous thrombosis. Percutaneous coil embolization with venous stenting can be an effective treatment in these circumstances.

\section{REFERENCES}

1 Parry DJ, Kessel D, Scott DJA. Simplifying the internal iliac artery aneurysm. Ann R Coll Surg Eng 2001;83:302-8
2 Su WT, Goldman KA, Riles TS, Rosen R. Deep venous thrombosis with pulmonary embolus after selective embolization of an internal iliac artery aneurysm. J Vasc Surg 1996;23:152-5

3 Secil M, Sarisoy HT, Hazan E, Goktay AY. Iliac artery aneurysm presenting with lower extremity deep vein thrombosis. J Emerg Med 2003;24:65-7

4 Hollis HW Jr, Luethke JM, Yakes WF, Beitler AL. Percutaneous embolization of an internal iliac artery aneurysm: technical considerations and literature review. Vasc Intervent Radiol 1994;5:449-57

5 Rosenthal D, Matsuura JH, Jerius H, Clark MD. Iliofemoral venous thrombosis caused by compression of an internal iliac artery aneurysm: a minimally invasive treatment. J Endovasc Surg 1998;5:142-5

\section{Crohn's disease and discitis}

\author{
S Kavia BSc MRCSEd J M Gilbert MS FRCS
}

$J$ S Soc Med 2004;97:484-485

CLINICAL SECTION, 17 OCTOBER 2002

Abscess formation, from deep fissuring of the bowel wall or suppuration within lymph nodes and local fistulation, develops in as many as $20 \%$ of patients with Crohn's disease. ${ }^{1}$ Several cases of vertebral and paravertebral infection have been described in association with enteric fistulas. Isolated discitis, however, has been little reported.

\section{CASE HISTORY}

A man of 58 consulted his general practitioner because of abdominal pain, diarrhoea and weight loss. An abdominal X-ray had shown partial obstruction of the large bowel and the possibility of air within the bowel wall. After admission to hospital he developed peritonitis from perforation of the descending colon and underwent left hemicolectomy, loop ileostomy and splenectomy (the inflamed colon being adherent to the spleen). Postoperatively he did well until the onset of high fever and leucocytosis due to an intra-abdominal collection. Percutanous drainage of this collection resulted in a chronic colocutanous fistula, specimens from which grew pseudomonas species. The fistula improved and he was discharged from hospital. A few weeks later, however, he had to be readmitted with recurrence of his intraabdominal collection and the fistula. He also had severe back pain. Again the fistula grew pseudomonas species. A sinogram showed a possible connection to bowel at the

Department of Surgery, Wexham Park Hospital, Slough SL2 4HL, UK

Correspondence to: Dr S Kavia, Flat One, Kendal Court, Shoot Up Hill, London NW2 3PD, UK

E-mail: skavia@blueyonder.co.uk 